\title{
A priori estimates for solutions of FitzHugh-Rinzel system
}

\author{
Monica De Angelis [
}

Received: 9 March 2021 / Accepted: 1 February 2022 / Published online: 1 March 2022

(C) The Author(s) 2022, corrected publication 2022

\begin{abstract}
The FitzHugh-Rinzel system is able to describe some biophysical phenomena, such as bursting oscillations, and the study of its solutions can help to better understand several behaviours of the complex dynamics of biological systems. We express the solutions by means of an integral equation involving the fundamental solution $H(x, t)$ related to a non linear integro-differential equation. Properties of $H(x, t)$ allow us to obtain a priori estimates for solutions determined in the whole space, showing both the influence of the initial data and the source term.
\end{abstract}

Keywords FitzHugh-Rinzel model $\cdot$ Fundamental solution $\cdot$ Laplace transform

\section{Introduction}

Mathematical biophysics models, such as the FitzHugh-Nagumo system (FHN), play an important role in studying the nervous system, as they can help describe biophysical phenomena that are relevant to neuronal excitability.

The FHN consists of two differential equations that model several engineering applications and there

\section{De Angelis $(\bowtie)$}

Department of Mathematics and Applications "R.

Caccioppoli", University of Naples Federico II, Via

Cinthia 26, 80126 Naples, Italy

e-mail: modeange@unina.it exist many scientific results and an extensive bibliography in regard [1-7]. However, it has been noted that only using a reset or adding noise, it is possible to evaluate bursting phenomena. This phenomenon occurs in a number of different cell types and it consists of a behaviour characterized by brief bursts of oscillatory activity alternating with periods of quiescence during which the membrane potential changes only slowly [8].

Bursting phenomena are becoming more and more important and their studies are increasing in many scientific fields (see, f.i. [9] and references therein). For example, in the restoration of synaptic connections, it appears that some nanoscale memristor devices have the potential to reproduce the behavior of a biological synapse $[10,11]$. This will lead in the future, especially in case of traumatic injuries, to the introduction of electronic synapses to directly connect neurons.

A model that seems to be more mathematically appropriate for incorporating nerve cell bursting phenomena is the FitzHugh-Rinzel model (FHR). It derives from FitzHugh-Nagumo and, differently from FHN, consists of three equations just to insert slow modulation of the current [1, 12-15]. Indeed, bursting oscillations can be characterized by a system variable that periodically changes from an active phase of rapid spike oscillations to a silent phase.

As for the FHR model, the following system is considered: 


$$
\left\{\begin{array}{l}
\frac{\partial u}{\partial t}=D \frac{\partial^{2} u}{\partial x^{2}}-w+y+f(u) \\
\frac{\partial w}{\partial t}=\varepsilon(-\beta w+c+u) \\
\frac{\partial y}{\partial t}=\delta(-u+h-d y)
\end{array}\right.
$$

where

$$
f(u)=u(a-u)(u-1) \quad(0<a<1),
$$

and terms $\beta, c, d, h, \varepsilon, \delta$ are positive constants that characterize the model's kinetics. The second order term with $D>0$ can be associated to the axial current in the axon, and it derives from the Hodgkin- Huxley theory for nerve membranes. Indeed, if $b$ represents the axon diameter and $r_{i}$ is the resistivity, the spatial variation in the potential $V$ gives the term $\left(b / 4 r_{i}\right) V_{x x}$ from which term $D u_{x x}$ derives (see f.i. [16]), and in [9] an analysis on contribution due to this term has been developed. Furthermore, when the fast variable $u$ simulates the membrane potential of a nerve cell, while the slow variable $w$ and the super-slow variable $y$ determine the corresponding number densities of ions, the model (1) simulates the propagation of impulses from one neuron to another, and studies on solutions can help in testing the responses of the various models in neuroscience.

Several methods have been developed to find exact solutions related to partial differential equations and an extensive bibliography on the study of analytical behaviors exists (see,f.i [17-23]). The aim of this paper is to determine a priori estimates for the FHR solution by means of suitable properties of the fundamental solution $H(x, t)$, showing how the effects due to the initial perturbation are vanishing when $t$ tends to infinity, and simultaneously, as time increases, the effect of the nonlinear source remains bounded.

The paper is organized as follows: in Sect. 2 we define the mathematical problem and report some of the results already proved in [9], as well as other results well known in literature. In Sect. 3, some properties related to the fundamental solution $H(x, t)$ are obtained and, in a subsection, some relationships on convolutions which characterize the explicit solution, are highlighted. In Sect. 4, estimates on convolution are proved and in Sect. 5, the solution is expressed by means of these particular convolution integrals. Finally, in Sect. 6, a priori estimates are showed.

\section{Mathematical considerations}

Indicating by $T$ an arbitrary positive constant, let us consider the set:

$$
\Omega_{T}=\{(x, t): x \in \mathfrak{R}, 0<t \leq T\} .
$$

Moreover, if

$$
\begin{aligned}
u(x, 0) & =u_{0}, w(x, 0) \\
& =w_{0} y(x, 0) \\
& =y_{0},(x \in \mathfrak{R})
\end{aligned}
$$

represent the initial values, then from $(1)_{2,3}$, one deduces:

$\left\{\begin{array}{l}w=w_{0} e^{-\varepsilon \beta t}+\frac{c}{\beta}\left(1-e^{-\varepsilon \beta t}\right)+\varepsilon \int_{0}^{t} e^{-\varepsilon \beta(t-\tau)} u(x, \tau) d \tau \\ y=y_{0} e^{-\delta d t}+\frac{h}{d}\left(1-e^{-\delta d t}\right)-\delta \int_{0}^{t} e^{-\delta d(t-\tau)} u(x, \tau) d \tau .\end{array}\right.$

Besides, letting

$$
\begin{aligned}
& f(u)=-a u+\varphi(u) \\
& \text { with } \varphi(u)=u^{2}(a+1-u) \quad 0<a<1,
\end{aligned}
$$

system (1) becomes

$$
\left\{\begin{array}{l}
\frac{\partial u}{\partial t}=D \frac{\partial^{2} u}{\partial x^{2}}-a u-w+y+\varphi(u) \\
\frac{\partial w}{\partial t}=\varepsilon(-\beta w+c+u) \\
\frac{\partial y}{\partial t}=\delta(-u+h-d y)
\end{array}\right.
$$

and hence, when

$$
\begin{aligned}
F(x, t, u)= & \varphi(u)-w_{0}(x) e^{-\varepsilon \beta t}+y_{0}(x) e^{-\delta d t} \\
& -\frac{c}{\beta}\left(1-e^{-\varepsilon \beta t}\right)+\frac{h}{d}\left(1-e^{-\delta d t}\right)
\end{aligned}
$$

denotes the source term, problem (6) with initial conditions (3), can be modified into the following initial value problem $\mathcal{P}$ : 
$\left\{\begin{array}{l}u_{t}-D u_{x x}+a u+\int_{0}^{t}\left[\varepsilon e^{-\varepsilon \beta(t-\tau)}+\delta e^{-\delta d(t-\tau)}\right] u(x, \tau) d \tau=F(x, t, u) \\ u(x, 0)=u_{0}(x) \quad x \in \mathfrak{R} .\end{array}\right.$

In order to determine the solution of problem (8), let us consider the following functions: (b) for fixed $t>0, H$ and its derivatives are vanishing exponentially fast as $|x|$ tends to infinity.

(c) In addition, it results $\lim _{t \rightarrow 0} H(x, t)=0$, for any fixed $\eta>0$, uniformly for all $|x| \geq \eta$.

To obtain results of existence and uniqueness for the problem (8), the theorem of fixed point can be applied

$$
\begin{aligned}
H_{1}(x, t)= & \frac{e^{-\frac{x^{2}}{4 D t}}}{2 \sqrt{\pi D t}} e^{-a t} \\
& \left.-\frac{1}{2} \int_{0}^{t} \frac{e^{-\frac{x^{2}}{4 D y}-a y}}{\sqrt{t-y}} \frac{\sqrt{\varepsilon} e^{-\beta \varepsilon(t-y)}}{\sqrt{\pi D}} J_{1}(2 \sqrt{\varepsilon y(t-y)})\right\} d y,
\end{aligned}
$$

$H_{2}=\int_{0}^{t} H_{1}(x, y) e^{-\delta d(t-y)} \sqrt{\frac{\delta y}{t-y}} J_{1}(2 \sqrt{\delta y(t-y)} d y$

where $J_{1}(z)$ is the Bessel function of first kind and order 1.

In [9] it has been verified that function $H(x, t)$ :

$H=H_{1}-H_{2}$

represents the fundamental solution of the parabolic operator

$L u \equiv u_{t}-D u_{x x}+a u+\int_{0}^{t}\left[\varepsilon e^{-\varepsilon \beta(t-\tau)}+\delta e^{-\delta d(t-\tau)}\right] u(x, \tau) d \tau$,

and the following theorem has been proved:

Theorem 1 In the half-plane $\mathfrak{R} e s>\max (-a,-\beta \varepsilon,-\delta d)$ the Laplace integral $\mathcal{L}_{t} H$ converges absolutely for all $x>0$, and it results:

$\mathcal{L}_{t} H=\frac{1}{\sqrt{D}} \frac{e^{-\frac{|x|}{\sqrt{D}} \sigma}}{2 \sigma}$

where

$\sigma^{2}=s+a+\frac{\delta}{s+\delta d}+\frac{\varepsilon}{s+\beta \varepsilon}$.

Moreover, function $\mathrm{H}(\mathrm{x}, \mathrm{t})$ satisfies some properties typical of the fundamental solution of heat equation, such as:

(a) $H(x, t) \in C^{\infty}, t>0, \quad x \in \Re$, and therefore, also according to [24], for initial term and source function we shall admit:

Assumption A Initial data $u_{0}$ is continuously differentiable and bounded together with its first derivative. The source term $F(x, t, u)$ is defined and continuous on the following set:

$Z=\left\{(x, t, u):(x, t) \in \Omega_{T},-\infty<u<\infty\right\}$.

Besides, for each $K>0$ and $|u|<K, F(x, t, u)$ is uniformly Lipschitz

continuous in $(x, t)$ for each compact set of $\Omega_{T}$ and it is bounded for bounded $u$.

Then, for all $\left(u_{1}, u_{2}\right)$, there exists a positive constant $W_{F}$ such that:

$\left|F\left(x, t, u_{1}\right)-F\left(x, t, u_{2}\right)\right| \leq W_{F}\left|u_{1}-u_{2}\right|$.

As a consequence, when the fundamental solution $H(x, t)$ and source function $F(x, t, u)$ satisfy theorem 1 and Assumption A, respectively, indicating by $u(x, t)$ a solution of problem $\mathcal{P}$, then $u$ assumes this form:

$$
\begin{aligned}
u(x, t)= & \int_{\mathfrak{R}} H(x-\xi, t) u_{0}(\xi) d \xi \\
& +\int_{0}^{t} d \tau \int_{\mathfrak{R}} H(x-\xi, t-\tau) F[\xi, \tau, u(\xi, \tau)] d \xi .
\end{aligned}
$$

On the other hand, if $u(x, t)$ is a continuous and bounded solution of (17), it is possible to prove that $u$ satisfies (8).

Consequently, it is possible to conclude that 
Theorem 2 Initial value problem (8) admits a unique solution only if (17) admits a unique continuous and bounded solution.

Besides, by means of fixed point theorem, (and extensive proofs can be found, f.i., in [24-29]), it is possible to prove the following theorem:

Theorem 3 When Assumption A is satisfied, then the initial value problem (8) admits a unique regular solution $u(x, t)$ in $\Omega_{T}$.

In this case, taking into account the source term $F(x, t)$ defined in (7), solution (17) assumes the following form: three positive functions, then the following theorem holds:

Theorem 4 The solution function H defined in (11) satisfies the following estimate:

$$
\begin{gathered}
|H| \leq \frac{e^{-\frac{x^{2}}{4 D t}}}{2 \sqrt{\pi D t}}\left[e^{-a t}+t \varepsilon A(t)+\delta t\left(1+\frac{\varepsilon t}{|a-\beta \varepsilon|}\right)\right. \\
\left.B(t)+\frac{\varepsilon t}{|a-\varepsilon \beta|} C(t)\right]
\end{gathered}
$$

Proof Since

$\left|J_{1}(2 \sqrt{\varepsilon y(t-y)})\right| \leq \sqrt{\varepsilon y(t-y)} \quad(y \leq t)$

$$
\begin{aligned}
u(x, t)= & \int_{0}^{t} d \tau \int_{\mathfrak{R}} H(x-\xi, t-\tau) \varphi[\xi, \tau, u(\xi, \tau)] d \xi \\
& +\left(\frac{h}{d}-\frac{c}{\beta}\right) \int_{0}^{t} d \tau \int_{\mathfrak{R}} H(x-\xi, t-\tau) d \xi+\frac{c}{\beta} \int_{0}^{t} e^{-\beta \varepsilon \tau} d \tau \int_{\mathfrak{R}} H(x-\xi, t-\tau) d \xi \\
& -\int_{0}^{t} e^{-\beta \varepsilon \tau} d \tau \int_{\mathfrak{R}} H(x-\xi, t-\tau) w_{0}(\xi) d \xi-\frac{h}{d} \int_{0}^{t} e^{-\delta d \tau} d \tau \int_{\mathfrak{R}} H(x-\xi, t-\tau) d \xi \\
& +\int_{0}^{t} e^{-\delta d \tau} d \tau \int_{\mathfrak{R}} H(x-\xi, t-\tau) y_{0}(\xi) d \xi+\int_{\mathfrak{R}} H(x-\xi, t) u_{0}(\xi) d \xi
\end{aligned}
$$

and this formula, together with relations (4), allows us to determine also $v(x, t)$ and $y(x, t)$ in terms of the data.

\section{Some properties related to $H(x, t)$}

In order to obtain a priori estimates and asymptotic effects, some properties related to the fundamental solution $H$ need to be evaluated.

More precisely, formula (18) shows the need to evaluate the convolution of the fundamental solution $H$ with respect to time and space.

Consequently, this section will include a first part where two theorems involving some properties related to $H(x, t)$ are showed, and a subsection where some premises allowing to prove properties related to convolution integrals, will be stated.

Let us start indicating by

$A(t)=\frac{e^{-\beta \varepsilon t}-e^{-a t}}{a-\beta \varepsilon} ; \quad B(t)=\frac{e^{-\delta d t}-e^{-a t}}{a-\delta d} ; \quad C(t)=\frac{e^{-\delta d t}-\overline{e^{-\beta \varepsilon t}}}{\beta \varepsilon-\delta d}$ from (9) it results:

$\left|H_{1}(x, t)\right| \leq \frac{e^{-\frac{x^{2}}{4 D t}}}{2 \sqrt{\pi D t}}\left[e^{-a t}+\varepsilon t \int_{0}^{t} e^{-a y} e^{-\beta \varepsilon(t-y)} d y\right]$

and hence:

$\left|H_{1}(x, t)\right| \leq \frac{e^{-\frac{x^{2}}{4 D t}}}{2 \sqrt{\pi D t}}\left[e^{-a t}+\varepsilon t \frac{e^{-\beta \varepsilon t}-e^{-a t}}{a-\varepsilon \beta}\right]$.

Moreover, from (10) and by means of (22), it results:

$\left|H_{2}\right| \leq \int_{0}^{t} \frac{e^{-\frac{x^{2}}{4 D y}}}{2 \sqrt{\pi D y}}\left[\begin{array}{ll}e^{-a y}+\varepsilon y & \frac{e^{-\beta \varepsilon y}-e^{-a y}}{a-\varepsilon \beta}\end{array}\right] e^{-\delta d(t-y)} \delta y d y$.

Consequently one obtains: 


$$
\begin{aligned}
\left|H_{2}\right| & \leq \frac{\delta t e^{-\frac{x^{2}}{4 D t}}}{2 \sqrt{\pi D t}}\left[\frac{e^{-\delta d t}-e^{-a t}}{a-\delta d}\left(1+\frac{\varepsilon t}{|a-\varepsilon \beta|}\right)\right. \\
+ & \left.\frac{\varepsilon t}{|a-\varepsilon \beta|} \frac{e^{-\delta d t}-e^{-\beta \varepsilon t}}{\beta \varepsilon-\delta d}\right]
\end{aligned}
$$

Hence, according to (11), for (22) and (23), theorem holds.

Now, let us introduce as $I_{0}$ the modified Bessel function of the first kind and order 0 , and let

$l=\min (a, \beta \varepsilon), \quad q=\min \{a, \beta \varepsilon, \delta d\}$,

$\lambda(t) \equiv 1+\pi t(\sqrt{\varepsilon}+\sqrt{\delta}+\pi t \sqrt{\delta \varepsilon})$.

The following theorem holds:

Theorem 5 The fundamental solution $H(x, t)$ defined in (11) satisfies the following estimates:

$$
\begin{aligned}
& \int_{\mathfrak{R}}|H(x-\xi, t)| d \xi \leq e^{-a t}+\sqrt{\varepsilon} \pi t e^{-\frac{\beta \varepsilon+a}{2} t} I_{0}\left(\frac{\beta \varepsilon-a}{2} t\right) \\
& +\sqrt{\delta} \pi t\left[e^{-\frac{\delta d+a}{2} t} I_{0}\left(\frac{\delta d-a}{2} t\right)+\sqrt{\varepsilon} \pi t e^{-\frac{\delta d+l}{2} t} I_{0}\left(\frac{\delta d-l}{2} t\right)\right]
\end{aligned}
$$

$\int_{\mathfrak{R}}|H(x-\xi, t)| d \xi \leq \lambda(t) e^{-q t}$

Besides, indicating by

$$
\begin{aligned}
S= & 1 / a+\sqrt{\varepsilon} \pi \frac{a+\beta \varepsilon}{2(a \beta \varepsilon)^{3 / 2}} \\
& +\sqrt{\delta} \pi\left[\frac{\delta d+a}{(a \delta d)^{3 / 2}}+3 \pi \sqrt{\varepsilon} \frac{\delta^{2} d^{2}+l^{2}}{4(l \delta d)^{5 / 2}}\right],
\end{aligned}
$$

one has:

$\int_{0}^{t} d \tau \int_{\Re}|H(x-\xi, t-\tau)| d \xi \leq S$.

Proof Considering that

$H=H_{1}-H_{2}$,

we will firstly focus on the integral involving $H_{1}$, and then on that involving $\mathrm{H}_{2}$.

Since it results:
$\int_{\Re} e^{-\frac{x^{2}}{4 D t}} d x=2 \sqrt{\pi D t} ; \quad\left|J_{1}(z)\right| \leq 1$,

from (9) one obtains:

$$
\int_{\mathfrak{R}}\left|H_{1}(x, t)\right| d x \leq e^{-a t}+\sqrt{\varepsilon} \int_{0}^{t} e^{-\beta \varepsilon(t-y)} e^{-a y} \frac{\sqrt{y}}{\sqrt{t-y}} d y
$$

with

$$
\begin{aligned}
& \int_{0}^{t} e^{-\beta \varepsilon(t-y)} e^{-a y} \sqrt{\frac{y}{t-y}} d y \\
& =-\int_{0}^{t} e^{-\beta \varepsilon(t-y)} e^{-a y}(t / 2-y) \frac{d y}{\sqrt{y(t-y)}}+ \\
& +\int_{0}^{t} e^{-\beta \varepsilon(t-y)} e^{-a y} \frac{t / 2 d y}{\sqrt{y(t-y)}} .
\end{aligned}
$$

Now, taking into account that

$$
\int_{0}^{2 b} e^{-s y}(b-y) \frac{1}{\sqrt{2 b y-y^{2}}} d y=\pi b e^{-s b} I_{1}(s b)
$$

and

$\int_{0}^{2 b} e^{-s y} \frac{1}{\sqrt{2 b y-y^{2}}} d y=\pi e^{-s b} I_{0}(s b)$

for $b=t / 2$ and $s=a-\beta \varepsilon$, one has:

$$
\begin{aligned}
& \int_{0}^{t} e^{-\beta \varepsilon(t-y)} e^{-a y} \sqrt{\frac{y}{t-y}} d y \\
& =\frac{\pi t}{2}\left[e^{-\frac{a-\beta \varepsilon}{2} t}\left(I_{0}\left(\frac{a-\beta \varepsilon}{2} t\right)-I_{1}\left(\frac{a-\beta \varepsilon}{2} t\right)\right)\right] .
\end{aligned}
$$

Consequently, as for

$$
I_{1}(-z)=-I_{1}(z) \quad I_{0}(z)=I_{0}(-z) \quad I_{1}(|z|) \leq I_{0}(|z|),
$$

it results:

$\int_{\mathfrak{R}}\left|H_{1}(x-\xi, t)\right| d \xi \leq e^{-a t}+\sqrt{\varepsilon} \pi t e^{-\frac{a+\beta \varepsilon}{2} t} I_{0}\left(\frac{\beta \varepsilon-a}{2} t\right)$.

Now, being $I_{0}(|z|)<e^{|z|}$, from (36) one deduces that

$\int_{\Re}\left|H_{1}(x-\xi, t)\right| d \xi \leq e^{-a t}+\sqrt{\varepsilon} \pi t e^{-l t}$

where $l$ is defined in $(24)_{1}$.

As for function $\mathrm{H}_{2}$, taking into account that $\left|J_{1}\right| \leq 1$, from (10) and by means of (37), it results: 
$\int_{\mathfrak{R}}\left|H_{2}(x-\xi, t)\right| d \xi \leq \sqrt{\delta} \int_{0}^{t}\left(e^{-a y}+\sqrt{\varepsilon} \pi y e^{-l y}\right) e^{-\delta d(t-y)} \sqrt{\frac{y}{t-y}} d y$.

Hence, returning to the previous reasoning, one obtains:

$$
\begin{aligned}
& \int_{\mathfrak{R}}\left|H_{2}\right| \leq \sqrt{\delta} \pi t\left[e^{-\frac{\delta d+a}{2} t} I_{0}\left(\frac{\delta d-a}{2} t\right)\right. \\
& \left.+\sqrt{\varepsilon} \pi t e^{-\frac{\delta d+l}{2} t} I_{0}\left(\frac{\delta d-l}{2} t\right)\right]
\end{aligned}
$$

from which, along with (36), (26) is proved.

Moreover, from (38), an inequality analogous to (37) can be obtained. In this way, according to (30), (27) follows, too.

Lastly, since it results

$$
\int_{0}^{\infty} e^{-p t} t I_{0}(b t) d t=p\left(\sqrt{p^{2}-b^{2}}\right)^{-3} \quad \operatorname{Re} p>|\operatorname{Re} b|
$$

$$
\begin{aligned}
\int_{0}^{\infty} e^{-p t} t^{2} I_{0}(b t) d t= & \left(\sqrt{p^{2}-b^{2}}\right)^{-3 / 2} \\
& \left(\frac{3 p^{2}}{p^{2}-b^{2}}-1\right) \quad \text { Re } p>|\operatorname{Re} b|,
\end{aligned}
$$

from (36) and (38), property (29) can be proved.

3.1 Premises on convolution integrals referring to the solution

In order to determine the estimates related to the solution, it is necessary to highlight every convolution integrals that characterize the solution itself. Therefore, in this subsection convolutions $K_{\delta}$ and $H_{\delta}$ will be introduced and, by means of them, solution $u(x, t)$ will be expressed (Formula 52).

Hence, let us consider

$$
K_{\delta}(x, t) \equiv \int_{0}^{t} e^{-\delta d(t-y)} H_{1}(x, y) J_{0}(2 \sqrt{\delta y(t-y)}) d y
$$

and let

$g_{1}(x, t) * g_{2}(x, t)=\int_{0}^{t} g_{1}(x, t-\tau) g_{2}(x, \tau) d \tau$ be the convolution with respect to $t$.

In [9] it has been proved that:

$e^{-\delta d t} * H=K_{\delta}$

and

$e^{-\varepsilon \beta t} * H=K_{\delta}+(\delta d-\varepsilon \beta) e^{-\beta \varepsilon t} * K_{\delta}$.

Now, denoting by

$H_{\delta}=\int_{0}^{t} e^{-\varepsilon \beta(t-\tau)} d \tau \int_{0}^{\tau} H_{1}(x, y) e^{-\delta d(\tau-y)} J_{0}(2 \sqrt{\delta y(\tau-y)}) d y$

it results:

$e^{-\beta \varepsilon t} * K_{\delta}=H_{\delta}$,

and as a consequence, from (44), one one:

$e^{-\varepsilon \beta t} * H=K_{\delta}+(\delta d-\varepsilon \beta) H_{\delta}$.

Moreover, let us denote by

$g_{1}(x, t) \diamond g_{2}(x, t)=\int_{\mathfrak{R}} f_{1}(\xi, t) g_{2}(x-\xi, t) d \xi$

the convolution with respect to the space, and

$H \otimes F=\int_{0}^{t} d \tau \int_{\Re} H(x-\xi, t-\tau) F[\xi, \tau, u(\xi, \tau)] d \xi$.

Since (43) and (47), it results:

$$
\left\{\begin{array}{l}
H \otimes e^{-\delta d t}=\int_{\Re} K_{\delta}(\xi, t) d \xi, \\
H \otimes e^{-\beta \varepsilon t}=\int_{\mathfrak{R}}\left[K_{\delta}+(\delta d-\varepsilon \beta) H_{\delta}\right] d \xi
\end{array}\right.
$$

and

$$
\left\{\begin{array}{l}
H \otimes\left(y_{0}(x) e^{-\delta d t}\right)=y_{0} \diamond K_{\delta} \\
H \otimes\left(w_{0}(x) e^{-\beta \varepsilon t}\right)=w_{0} \diamond\left[K_{\delta}+(\delta d-\varepsilon \beta) H_{\delta}\right] .
\end{array}\right.
$$

Consequently, given (18), we get: 


$$
\begin{aligned}
& u(x, t)=H \diamond u_{0}(x)+K_{\delta} \diamond\left(y_{0}(x)-w_{0}(x)\right)+H \otimes \varphi(u) \\
& +(\varepsilon \beta-\delta d) H_{\delta} \diamond w_{0}(x)+\frac{c}{\beta} H_{\delta} \diamond(\delta d-\varepsilon \beta) \\
& +H \otimes\left(\frac{h}{d}-\frac{c}{\beta}\right)+K_{\delta} \diamond\left(\frac{c}{\beta}-\frac{h}{d}\right)
\end{aligned}
$$

and this formula explicitly shows all the convolutions involved in the solution $u(x, t)$.

\section{On convolutions involving functions $K_{\delta}$ and $H_{\delta}$}

Formula (52) shows that an analysis of the solution directly implies estimates on both $H(x, t)$ and on functions $K_{\delta}, H_{\delta}$, defined in (41) and (45).

For this, let us consider $A(t), B(t), C(t), \lambda(t)$ defined in (19) and (25), respectively. Moreover, let

$E(t)=\frac{e^{-q t}-e^{-\delta d t}}{\delta d-q} \quad L(t)=\frac{e^{-q t}-e^{-\beta \varepsilon t}}{\beta \varepsilon-q}$

with $q$ defined by $(24)_{2}$.

In addition,

$M=\frac{1}{|\delta d-q| \delta q d}\left[q+\delta d+\pi(\sqrt{\varepsilon}+\sqrt{\delta})\left(\frac{q^{2}+\delta^{2} d^{2}}{\delta d q}\right)+2 \pi^{2} \sqrt{\delta \varepsilon}\left(\frac{q^{3}+\delta^{3} d^{3}}{(q \delta d)^{2}}\right)\right]$

$N=\frac{1}{|\beta \varepsilon-q| q \beta \varepsilon}\left[q+\beta \varepsilon+\pi(\sqrt{\varepsilon}+\sqrt{\delta})\left(\frac{q^{2}+\beta^{2} \varepsilon^{2}}{\beta \varepsilon q}\right)+2 \pi^{2} \sqrt{\delta \varepsilon}\left(\frac{q^{3}+\beta^{3} \varepsilon^{3}}{(q \beta d)^{2}}\right)\right]$

$g(t)=\frac{\lambda(t)}{|\beta \varepsilon-\delta d|}[E(t)+L(t)]$

$h(t)=\frac{\lambda(t)}{(\varepsilon \beta-\delta d)^{2}}[L(t)+(1+t(\delta d-\varepsilon \beta] E(t)$.

The following theorems hold:

Theorem 6 Function $K_{\delta}(x, t)$ defined in (41) satisfies the following estimates:

$\int_{\Re}\left|K_{\delta}(x, t)\right| \leq \lambda(t) E(t) ;$

$\int_{0}^{t} d \tau \int_{\Re}\left|K_{\delta}(x, \tau)\right| d x \leq M$. $\int_{0}^{t} e^{-\delta d \tau} d \tau \int_{\mathfrak{R}}\left|K_{\delta}(x, t-\tau)\right| d x \leq t \lambda(t) E(t)$

Proof By means of (43) and property (27) on $\int_{\mathfrak{R}}|H(\xi, t)| d \xi$, inequality (58) follows.

By this estimate, according to (25), and taking into account that

$$
\begin{gathered}
\int_{0}^{t} y e^{-\alpha y} \leq 1 / \alpha^{2} ; \quad \int_{0}^{t} y^{2} e^{-\alpha y} \leq 2 / \alpha^{3} \\
(t>0, \quad \alpha>0),
\end{gathered}
$$

(59) holds, too.

Moreover, because of (43), it results

$e^{-\delta d t} * K_{\delta}=e^{-\delta d t} * H * e^{-\delta d t}=\left(t e^{-\delta d t}\right) * H$

and inequality (60) follows.

Theorem 7 Referring to (45), function $H_{\delta}(x, t)$ satisfies the inequalities below: 
$\int_{\mathfrak{R}}\left|H_{\delta}(x, t)\right| d x=\int_{0}^{t} e^{-\beta \varepsilon \tau} d \tau \int_{\Re}\left|K_{\delta}(x, t-\tau)\right| d x$

with, since (43), it results:

$e^{-\beta \varepsilon t} * K_{\delta}=e^{-\beta \varepsilon t} * H * e^{-\delta d t}=C(t) * H(x, t)$

where $C(t)$ is defined in $(19)_{3}$. Hence, since (27), inequality (63) holds.

Consequently, also (64) follows.

Estimate (65) is proved by means of

$e^{-\delta d t} * H_{\delta}=e^{-\delta d t} * K_{\delta} * e^{-\beta \varepsilon t}=\left(t e^{-\delta d t}\right) * e^{-\beta \varepsilon t} * H$.

Finally, taking into account that

$$
\begin{aligned}
u(x, t)= & \int_{0}^{t} d \tau \int_{\mathfrak{R}} H(x-\xi, t-\tau) \varphi[\xi, \tau, u(\xi, \tau)] d \xi \\
& +\left(\frac{h}{d}-\frac{c}{\beta}\right)\left[\int_{0}^{t} d \tau \int_{\mathfrak{R}} H(x-\xi, t-\tau) d \xi-\int_{\mathfrak{R}} K_{\delta}(x-\xi, t) d \xi\right] \\
& +\int_{\mathfrak{R}} K_{\delta}(x-\xi, t)\left[y_{0}(\xi)-w_{0}(\xi)\right] d \xi-(\delta d-\varepsilon \beta) \int_{\mathfrak{R}} H_{\delta}(x-\xi, t) w_{0}(\xi) d \xi \\
& +\frac{c}{\beta}(\delta d-\varepsilon \beta) \int_{\mathfrak{R}} H_{\delta}(x-\xi, t) d \xi+\int_{\mathfrak{R}} H(x-\xi, t) u_{0}(\xi) d \xi .
\end{aligned}
$$

\section{Analysis of solution}

In order to analyse functions $u(x, t), w(x, t)$, and $y(x, t)$, it appears necessary to make explicit the integrals of convolutions involving functions $H_{\delta}$ and $K_{\delta}$ whose estimates have been established in the previous section.

Therefore, since (52), by means of convolution properties, we get:

$$
\begin{aligned}
\int_{0}^{t} e^{-\beta \varepsilon(t-\tau)} u(x, \tau) d \tau= & \int_{\mathfrak{R}} K_{\delta}(x-\xi, t) u_{0}(\xi) d \xi \\
& +\int_{0}^{t} d \tau \int_{\mathfrak{R}} K_{\delta}(x-\xi, t-\tau)\left[\varphi[\xi, \tau, u(\xi, \tau)]+\frac{h}{d}-\frac{c}{\beta}\right] d \xi \\
& +(\delta d-\varepsilon \beta) \int_{0}^{t} d \tau \int_{\mathfrak{R}} H_{\delta}(x-\xi, t-\tau)\left[\varphi[\xi, \tau, u(\xi, \tau)]+\frac{h}{d}-\frac{c}{\beta}\right] d \xi \\
& +(\delta d-\varepsilon \beta) \int_{0}^{t} e^{-\beta \varepsilon(t-\tau)} d \tau \int_{\mathfrak{R}} H_{\delta}(x-\xi, \tau)\left[\frac{c}{\beta}-w_{0}(\xi)\right] d \xi \\
& +\int_{\mathfrak{R}} H_{\delta}(x-\xi, t)\left[y_{0}(\xi)-w_{0}(\xi)-\frac{h}{d}+\frac{c}{\beta}+(\delta d-\varepsilon \beta) u_{0}(\xi)\right] d \xi
\end{aligned}
$$


and

$$
\begin{aligned}
\int_{0}^{t} e^{-\delta d(t-\tau)} u(x, \tau) d \tau= & \int_{\mathfrak{R}} K_{\delta}(x-\xi, t) u_{0}(\xi) d \xi \\
& +\int_{0}^{t} d \tau \int_{\mathfrak{R}} K_{\delta}(x-\xi, t-\tau)\left[\varphi[\xi, \tau, u(\xi, \tau)]+\frac{h}{d}-\frac{c}{\beta}\right] d \xi \\
& +\int_{0}^{t} e^{-\delta d(t-\tau)} d \tau \int_{\mathfrak{R}} K_{\delta}(x-\xi, \tau)\left[y_{0}(\xi)-w_{0}(\xi)-\frac{h}{d}+\frac{c}{\beta}\right] d \xi \\
& +(\delta d-\varepsilon \beta) \int_{0}^{t} e^{-\delta d(t-\tau)} d \tau \int_{\mathfrak{R}} H_{\delta}(x-\xi, \tau)\left[\frac{c}{\beta}-w_{0}(\xi)\right] d \xi
\end{aligned}
$$

\section{Estimates of solution}

As for the analysis of solutions of the non linear reaction diffusion model, there exists a large bibliography. In particular in $[30,31]$ the existence of bounded solutions is proved.

Therefore, in the class of bounded solutions, let us assume initial data and function $\varphi(x, t, u)$ satisfy Assumption A, and let

$$
\begin{aligned}
\left\|u_{0}\right\|=\sup _{\mathfrak{R}}\left|u_{0}(x)\right|, \quad\left\|w_{0}\right\|=\sup _{\Re}\left|w_{0}(x)\right|, \\
\left\|y_{0}\right\|=\sup _{\mathfrak{R}}\left|y_{0}(x)\right|, \\
\|u\|=\sup _{\Omega_{T}}\left|u(x, t) \quad\|\varphi\|=\sup _{Z}\right| \varphi(x, t, u) \mid
\end{aligned}
$$

with $\varphi$ defined in (5) and $Z$ defined in (15).

In order to give a priori estimates of the solution of FHR system, the following theorem is proved:

Theorem 8 If function $\varphi(x, t, u)$ and initial data $u_{o}(x), w_{o}(x), y_{o}(x)$ are compatible with Assumption $A$, then the problem (1)-(3) satisfies the following estimates:

$$
\begin{aligned}
|u(x, t)| & \leq\left\|u_{0}(x)\right\| \lambda(t) e^{-q t}+\left(\|\varphi\|+\left|\frac{h}{d}-\frac{c}{\beta}\right|\right) S \\
& \left.+\left(\left\|y_{0}\right\|+\| w_{0}\right) \|+\left|\frac{h}{d}-\frac{c}{\beta}\right|\right) \lambda(t) E(t) \\
& +\left(\left\|w_{0}\right\|+\frac{c}{\beta}\right)(|\delta d-\varepsilon \beta|) g(t) ;
\end{aligned}
$$

$|w(x, t)| \leq\left\|w_{0}\right\| e^{-\beta \varepsilon t}+\frac{c}{\beta}+\varepsilon\left\|u_{0}\right\| \lambda(t) E(t)$

$$
\begin{aligned}
& +\varepsilon\left(\|\varphi\|+\left|\frac{h}{d}-\frac{c}{\beta}\right|\right)(2 M+N)+ \\
& \left.+\varepsilon \frac{|\delta d-\varepsilon \beta|}{|\delta d-q|}\left[\frac{c}{\beta}+\left\|w_{0}(x)\right\|\right]\right] \lambda(t)[C(t)+L(t)] \\
& +\varepsilon\left[\left\|y_{0}\right\|+\left\|w_{0}\right\|+\left|\frac{c}{\beta}-\frac{h}{d}\right|+|\delta d-\varepsilon \beta|\left\|u_{0}\right\|\right] g(t) ;
\end{aligned}
$$

$$
\begin{aligned}
|y(x, t)| & \leq\left\|y_{0}\right\| e^{-\delta d t}+\frac{h}{d}+\delta\left\|u_{0}\right\| \lambda(t) E(t)+ \\
& +\delta\left[\left\|y_{0}\right\|+\left\|w_{0}\right\|+\left|\frac{c}{\beta}-\frac{h}{d}\right|\right] t \lambda(t) E(t)+ \\
& \delta\left[\|\varphi\|+\left|\frac{h}{d}-\frac{c}{\beta}\right|\right] M+(\delta d-\varepsilon \beta)\left(\left\|w_{0}\right\|+\frac{c}{\beta}\right) h(t)
\end{aligned}
$$

where constants $q, S, M, N$ are introduced in $(24)_{2},(28),(54)$, and (55), respectively.

Besides, functions $C(t), \lambda(t), E(t), L(t), g(t), h(t)$ are defined in $(19)_{3}(25),(53)_{1,2},(56)$, and (57).

Proof According to (71) and by means of inequalities (27), (29), (58), and (63), estimate (74) follows.

As for inequalities (75) and (76), functions defined in (4) have to be considered.

More precisely, from (4) 1 and (72), taking into account inequalities (58), (59), (63), (64) and (66), estimate (75) is proved.

noindent Analogously, from (4) $)_{2}$ and (73), for (58)-(60) and (65), also (76) holds. 
Remark These estimates show that the solution of the FitzHugh-Rinzel system is bounded for all t. Besides, when $\mathrm{t}$ tends to infinity, the effect of the non linear term $\varphi(x, t)$ is bounded, while the effects of initial perturbances $u_{0}(x), w_{0}(x), y_{0}(x)$ are vanishing.

Acknowledgements The present work has been developed with the economic support of MIUR (Italian Ministry of University and Research) performing the activities of the project ARS01_00861 "Integrated collaborative systems for smart factory - ICOSAF". The paper has been performed under the auspices of G.N.F.M. of INdAM. The author is grateful to the anonymous reviewers for their comments and suggestions.

Funding Open access funding provided by Università degli Studi di Napoli Federico II within the CRUI-CARE Agreement.

\section{Declarations}

Conflict of interest The author declares that she has no conflict of interest.

Open Access This article is licensed under a Creative Commons Attribution 4.0 International License, which permits use, sharing, adaptation, distribution and reproduction in any medium or format, as long as you give appropriate credit to the original author(s) and the source, provide a link to the Creative Commons licence, and indicate if changes were made. The images or other third party material in this article are included in the article's Creative Commons licence, unless indicated otherwise in a credit line to the material. If material is not included in the article's Creative Commons licence and your intended use is not permitted by statutory regulation or exceeds the permitted use, you will need to obtain permission directly from the copyright holder. To view a copy of this licence, visit http://creativecommons.org/licenses/by/4.0/.

\section{References}

1. Izhikevich EM (2007) Dynamical systems in neuroscience: the geometry of excitability and bursting. The MIT Press, London, p 397

2. Renno P, De Angelis M (2014) Asymptotic effects of boundary perturbations in excitable systems. Discret Contin Dyn Syst Ser B 19(7):2039-2045

3. De Angelis M (2021) A note on explicit solutions of FitzHugh-Rinzel system (2021) Nonlinear Dyn Syst Theory 21(4):360-366

4. Rionero S, Torcicollo I (2018) On the dynamics of a nonlinear reaction-diffusion duopoly model. Int $\mathrm{J}$ Non-Linear Mech 99:105-111

5. De Angelis M (2013) Asymptotic estimates related to an integro differential equation. Nonlinear Dyn Syst Theory 13(3):217-228
6. Gambino G, Lombardo MC, Rubino G, Sammartino M (2018) Pattern selection in the 2D FitzHugh-Nagumo model. Ric Mat 68:535-549

7. De Angelis M (2013) A priori estimates for excitable models. Meccanica 48(10):2491-2496

8. Keener JP, Sneyd J (1998) Mathematical physiology. Springer, New York (1998)

9. De Angelis F, De Angelis M(2020) On solutions to a FitzHughRinzel type model. Ric Mat https://doi.org/10.1007/ s11587-020-00483-y

10. Juzekaeva E, Nasretdinov A, Battistoni S, Berzina T, Iannotta S, Khazipov R, Erokhin V, Mukhtarov M (2019) Coupling cortical neurons through electronic memristive synapse. Adv Mater Technol 4:1800350

11. Corinto F, Lanza V, Ascoli A, Marco G (2011) Synchronization in networks of FitzHugh-Nagumo neurons with memristor synapses. In: IEEE 20th European conference on circuit theory and design (ECCTD). IEEE

12. Bertram R, Manish T, Butte J, Kiemel T, Sherman A (1995) Topological and phenomenological classification of bursting oscillations. Bull Math Biol 57(3):413

13. Wojcik J, Shilnikov A (2015) Voltage interval mappings for an elliptic bursting model in nonlinear dynamics new directions theoretical aspects, vol 12, pp 195-213. Springer, Berlin

14. Rinzel J, Troy WC (1982) Bursting phenomena in a simplified Oregonator flow system model. J Chem Phys 76:1775-1789

15. Rinzel J (1987) A formal classification of bursting mechanisms in excitable systems. Mathematical topics in population biology, morphogenesis and neurosciences. Lecture notes in biomathematics, vol 71, pp 267-281. Springer, Berlin

16. Murray JD (2003) Mathematical biology I. Springer, New York

17. De Angelis M (2018) On the transition from parabolicity to hyperbolicity for a nonlinear equation under Neumann boundary conditions. Meccanica 53(15):3651-3659

18. Li H, Guoa Y (2006) New exact solutions to the Fitzhugh Nagumo equation. Appl Math Comput 180(2):524-528

19. Fiore G, De Angelis M (2014) Diffusion effects in a superconductive model. Commun Pure Appl Anal 13(1):217-223 (2014)

20. De Angelis M (2019) A wave equation perturbed by viscous terms: fast and slow times diffusion effects in a Neumann problem. Ric Mat 68(1):237-252

21. Prinaria B, Demontis F, Li Sitai, Horikis TP (2018) Inverse scattering transform and soliton solutions for square matrix nonlinear Schrödinger equations with non-zero boundary conditions. Physica D 368:22-49

22. De Angelis M (2015) Mathematical contributions to the dynamics of the Josephson junctions: state of the art and open problems. Nonlinear Dyn Syst Theory 15(3):231-241

23. Kudryashov NK (2018) Asymptotic and exact solutions of the FitzHugh-Nagumo model. Regul Chaotic Dyn 23(2):152-160

24. Cannon JR (1984) The one-dimensional heat equation. Addison-Wesley Publishing Company, Boston

25. Renno P, De Angelis M (2014) On asymptotic effects of boundary perturbations in exponentially shaped Josephson junctions. Acta Appl Math 132:251-259

26. Fiore G, De Angelis M (2013) Existence and uniqueness of solutions of a class of third order dissipative problems with 
various boundary conditions describing the Josephson effect. J Math Anal Appl 404(2):477-490

27. D'Anna AG, Fiore, De Angelis M (2012) Existence and uniqueness for some 3rd order dissipative problems with various boundary conditions. Acta Appl Math 122(1)

28. Renno P, De Angelis M (2008) Existence, uniqueness and a priori estimates for a non linear integro-differential equation. Ric Mat 57:95-109

29. De Angelis M (2012) On exponentially shaped Josephson junctions. Acta Appl Math 122(1):179-189. https://doi.org/ 10.1007/s10440-012-9736-9
30. Smoller J (1994) Shock waves and reaction-diffusion equations, 2nd edn. Springer, New York

31. Rionero S (2021) Longtime behaviour and bursting frequency, via a simple formula, of FitzHugh-Rinzel neurons. Rend Fis Acc Lincei 32:857-867. https://doi.org/10. 1007/s12210-021-01023-y

Publisher's Note Springer Nature remains neutral with regard to jurisdictional claims in published maps and institutional affiliations. 